\title{
Functional Recovery of Cricket Giant Interneurons after Cercal Ablations
}

\author{
Tetsuya Matsuura ${ }^{1,3}$ and Masamichi Kanou ${ }^{2 *}$ \\ ${ }^{1}$ Department of Biology, Faculty of Science, Okayama University, Okayama, Japan \\ ${ }^{2}$ Department of Biology and Earth Sciences, Faculty of Science, \\ Ehime University, Matsuyama, Japan
}

\begin{abstract}
In order to explore the functional recovery of the cercal sensory system of the cricket Gryllus bimaculatus, changes in response properties of four air-motion sensitive giant interneurons (Gls 8-1, 9-1, 9-2 and 9-3) were investigated after partial sensory deprivations. Velocity thresholds, response magnitudes and response latencies of each $\mathrm{Gl}$ to a directional air current stimulus were investigated 21 days after ipsilateral or contralateral cercal ablations. The results were compared to those measured 1 day after the same treatment (Matsuura and Kanou, 1998) in order to specify the changes which occurred during the first 20 days. Each Gl showed a different pattern of change in responses according to the direction of stimulus air current; i.e. the changes observed were direction dependent regardless of whether they were compensational or not. Compensational changes in response magnitudes and/or response latencies were observed in Gls 8-1, 9-1 and 9-2 when air currents were applied from particular directions. As there was no regeneration of cercal filiform hairs, those changes must be caused by the changes in synaptic strength between Gls and particular sensory afferents associated with cercal filiform hairs. Such neural compensation must underlie the basis of behavioral compensation when the insects have damaged sensory apparatus.
\end{abstract}

\section{INTRODUCTION}

The nervous system of animals plays the most important role in making a behavioral response to a stimulus input. In most species of animals, especially in lower animals, the behavioral pattern to a particular stimulus is rigidly fixed. Therefore, the pattern of released behavior is one of the main clues to distinguish one species from others. This suggests that the nervous system of an animal is originally designed (or programmed) to make a particular output to a particular stimulus input, and the system is well known as an innate releasing mechanism (IRM; e.g. Tinbergen, 1969). In addition to such inflexibility, however, a nervous system sometimes shows flexible or plastic natures. The investigation of a neural plasticity is one of the most important subjects in current neuroethology and neurophysiology because it is the basis to understand higher functions of a nervous system such as memory and learning (Carew and Sahley, 1986; Thompson, 1986; Tsukahara, 1981; Kandel, 1979; Fetz and Baker, 1973). As a case study to explore the neural plasticity, we investigated the functional changes in central neurons of a cricket after a deprivation of cercal sensory inputs. Central compensation after the

\footnotetext{
* Corresponding author: Tel. +81-89-927-9631; FAX. +81-89-927-8909.

${ }^{3}$ Present address: Akita Laboratory, Japan Science and Technology Corporation, c/o Akita Research Institute of Brain and Blood Vessels, 6-10, Senshu-Kubota machi, Akita 010, Japan.
}

distortion or ablation of sensory inputs is generally observed in both invertebrate and vertebrate animals (Vardi and Camhi, 1982; Ito, 1982; Gonshor and Melvill-Jones, 1976). The cercal sensory system of a cricket is suited for this kind of analysis because response characteristics of each neural element have been investigated quantitatively (Kanou and Shimozawa, 1984; Shimozawa and Kanou, 1984a, b; Kanou, 1991, 1996).

Damages to sensory apparatus is a great disadvantage to animals for survival in natural environments because such damages generally reduce the amount of useful sensory information and change the related behaviors incorrectly. However, such incorrect behaviors are occasionally compensated after a certain time period. For example, cockroaches, which can recognize the position of predators from the air motion and make appropriate escape behaviors, often did not discriminate air puffs delivered to their left side from those delivered to their right side after removing one of a pair of cerci which bears a number of mechanosensitive filiform hairs. However, the left-right discrimination was partially recovered after 30 days (Vardi and Camhi, 1982). Similar behavioral compensation in wind-evoked escape was also observed in crickets (Gryllus bimaculatus) after partial sensory deprivations (Kanou et al., 1995). Such behavioral compensations must be supported by the changes occurred somewhere in the neural network because there was no regeneration of ablated filiform hairs during the recovery period. In the present study, we investigated the functional changes of 4 cricket Gls 
(Gls 8-1, 9-1, 9-2 and 9-3) after a unilateral cercal ablation. Velocity thresholds, response magnitudes and response latencies of each GI to an air current stimulus were measured 21 days after unilateral cercal ablation. The results were then compared to those measured 1 day after the same treatment (Matsuura and Kanou, 1998 and present study) in order to investigate whether a significant recovery (compensation) occurred during the first 20 days or not. Changes in the response properties of Gls, if any, must tell us a part of neural mechanisms underlying the basis of the behavioral compensation.

\section{MATERIALS AND METHODS}

\section{Animals}

Adult female crickets (Gryllus bimaculatus) reared in our laboratory were used. They were 1-2 weeks old after imaginal molt. The temperature of the culture room was kept at $28-30^{\circ} \mathrm{C}$ and the LD cycle was $14: 10$. Details were the same as in the previous paper (Kanou, 1996).

\section{Air current stimulation}

A wind-tunnel consisted of an acrylic tube equipped with a pair of push-pull driven loud speakers at both ends (Kanou and Shimozawa, 1984) was used for delivering an air current stimulus. Experimental setups and stimulating methods are the same as in the previous studies (Kanou, 1991, 1996).

Unidirectional air currents with peak velocities ranging from 0.095 $\mathrm{mm} / \mathrm{sec}$ to $300 \mathrm{~mm} / \mathrm{sec}$ were used. Direction of stimulus air current was changed by $30^{\circ}$ intervals in the horizontal plane (Fig. 1). At each stimulus direction, 10 successive air current stimuli with a particular peak velocity were applied to an insect in 5 second intervals. Details of stimulating procedures were the same as in the companion paper (Matsuura and Kanou, 1998).

\section{Neural activity recordings}

Responses of Gls in animals 21 days after ipsilateral or contralateral cercal ablation (21-day animal) were intracellulary recorded from axons in the right side connectives. After physiological investigations, the Gls were intracellulary marked with fluorescent dye Lucifer Yellow $\mathrm{CH}$ for a morphological identification. Details of the neural recording and intracellular marking procedures were the same as those in our companion paper (Matsuura and Kanou, 1998).

\section{Ablation experiments}

An ipsilateral or contralateral cercus was removed from the stump with a sharp razor blade. Special attention was paid not to leave any mechanosensitive filiform hairs. After the ablation, the insects were isolated in a transparent container $(20 \mathrm{~cm} \times 20 \mathrm{~cm} \times 25 \mathrm{~cm})$ with a lid off.

"Ipsilateral" or "contralateral" were defined to refer to the side of ventral nerve cord in which a Gl's axon ran (right side connectives). Crickets whose ipsilateral (right side) cercus was ablated were called $\mathrm{CCl}$ (contralateral cercus intact) animals. In the same way, contralateral (left side) cercus ablated crickets were called ICI (ipsilateral cercus intact) animals. Normal intact crickets were called BCl (both cerci intact) animals. On the other hand, crickets 21 (or 1) days after the cercal ablation were called "21-day (or 1-day) animals". Therefore, for example, crickets 21 days after the contralateral cercal ablation were expressed as "21-day ICl animals".

\section{Functional compensation}

By the unilateral cercal ablation, some of the response properties (velocity thresholds, response magnitudes or response latencies) of a Gl investigated in 1-day animals showed statistically significant change (t-test) from those in $\mathrm{BCl}$ animals in particular stimulus conditions (Matsuura and Kanou, 1998 and present study). However, some of them showed recovery 20 days after the investigation in 1-day animals. If such changes were statistically significant (t-test), they were assessed to be compensational.

\section{RESULTS}

Velocity thresholds, intensity-response relations and response latencies of each $\mathrm{Gl}$ were investigated in both 21-day $\mathrm{ICl}$ and $\mathrm{CCl}$ crickets by using unidirectional air currents. The results were compared to those measured in 1-day animals (Matsuura and Kanou, 1998; companion paper) in order to explore how the response properties changed during the first 20 days.

\section{GI 8-1 in ICl animals}

In 21-day ICI animals, velocity thresholds of Gl 8-1 were very similar to those in 1-day $\mathrm{ICI}$ animals regardless of the stimulus direction. Therefore, the receptive field of the $\mathrm{GI}$ in 21-day animals were almost identical to those of the 1-day animals (Fig. 1A). During the first 20 days, no significant change in threshold was detected in GI 8-1.

In $\mathrm{ICl}$ animals, there was no case in which response magnitude of $\mathrm{Gl} 8-1$ increased during the first 20 days period, i.e. most response magnitudes of the $\mathrm{Gl}$ decreased to some extent compared with those in 1-day $\mathrm{ICl}$ animals (Fig. 2). As most response magnitudes of GI 8-1 in 1-day $\mathrm{ICl}$ animals showed an increase compared to those in $\mathrm{BCl}$ animals (Fig. 2; Matsuura and Kanou, 1998), the response magnitudes in 21-day $\mathrm{ICl}$ animals were more similar to those in $\mathrm{BCl}$ animals than in 1-day ICl animals (except R60 and R90). Although most of the changes which occurred during the first 20 days seemed compensational, only the changes occurred to the air currents applied from R30, L150 and L90 directions were statistically significant $(P<0.05)$. When the air currents were applied from R60 and R90 directions, response magnitudes of GI 8-1 also showed a decrease as compared to those in 1-day animals. However, these changes were not compensational because the difference in response magnitudes between $\mathrm{BCl}$ and $\mathrm{ICl}$ animals became larger (Fig. 2).

Response latencies of GI 8-1 in 21-day ICl animals showed statistically significant changes only when the air current was applied from R30 direction (Fig. 3). However, the changes were not compensational. No statistically significant change in response latency was observed in GI 8-1 in ICl animals.

\section{GI 8-1 in $\mathbf{C C l}$ animals}

Like in 1-day CCl animals (Matsuura and Kanou, 1998), velocity thresholds of GI 8-1 in 21-day $\mathrm{CCl}$ animals were too high to specify the directional characteristics (Fig. 2). Therefore, any change in velocity thresholds was not ascertained in GI 8-1 in $\mathrm{CCl}$ animals during the initial 20 days.

In 1-day $\mathrm{CCl}$ animals, $\mathrm{Gl}$ 8-1 showed no response at all to the air currents applied from 0, R30, L60 and L30 direc- 

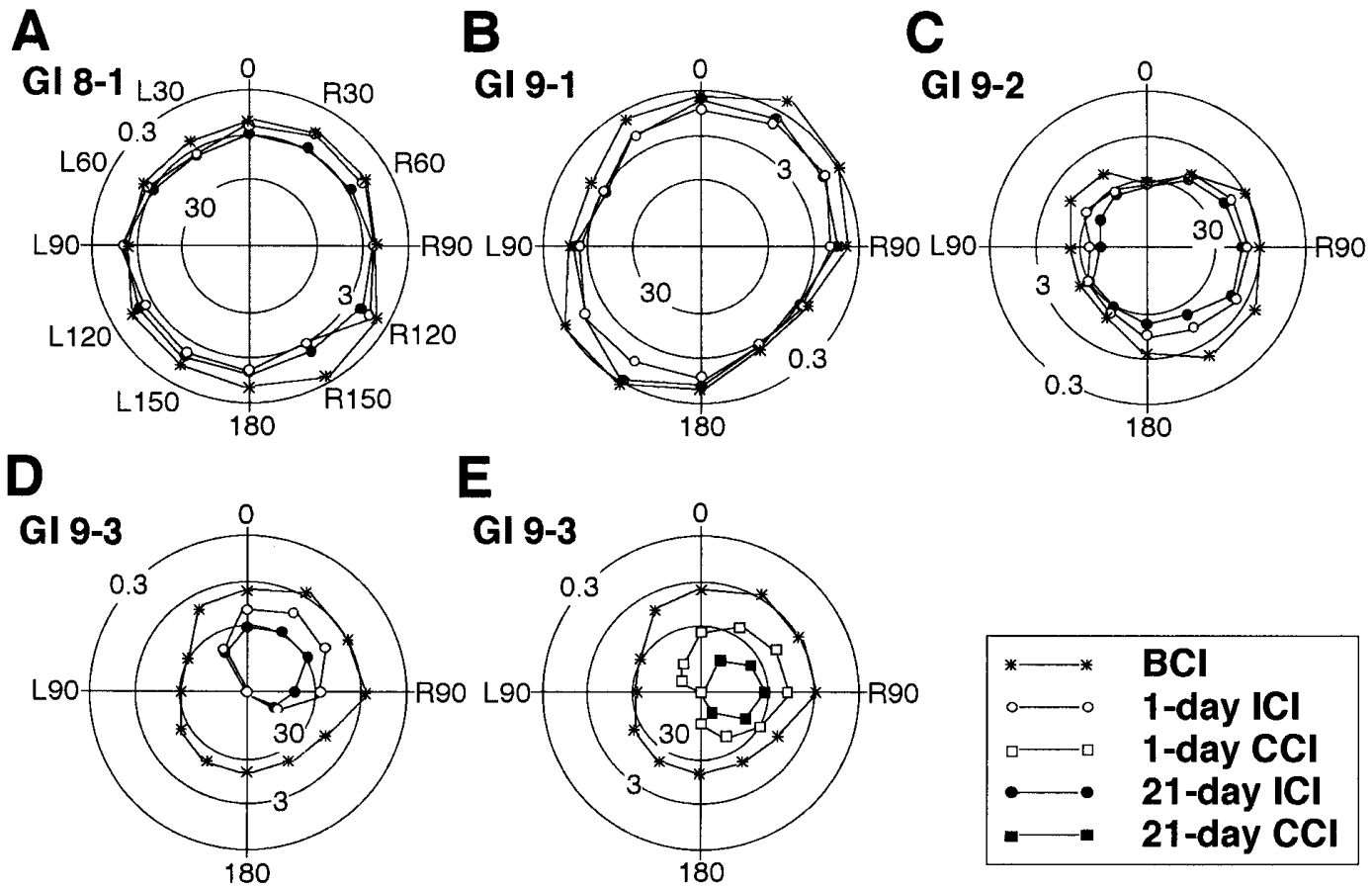

Fig. 1A-E. Receptive fields of $4 \mathrm{Gls}$, whose axons are running in the right side connective, are expressed with velocity thresholds. Velocity thresholds $(\mathrm{mm} / \mathrm{sec})$ of each $\mathrm{Gl}$ to an air current from different directions were plotted on polar coordinates in order to explore the changes after unilateral cercal ablations. (A) GI 8-1. (B) GI 9-1. (C) GI 9-2 . (D and E) GI 9-3. Crosses: BCl animals (data from Kanou, 1996). Open circles: 1-day ICl animals (data from Matsuura and Kanou, 1998). Filled circles: 21-day ICl animals. Open squares: 1-day CCl animals (data from Matsuura and Kanou, 1998). Filled squares: 21-day CCl animals. Velocity thresholds of Gls 8-1, 9-1 or 9-2 in CCl animals were not obtained. Between 1- and 21-day animals, no statistically significant compensational change in velocity thresholds was observed in any GI.

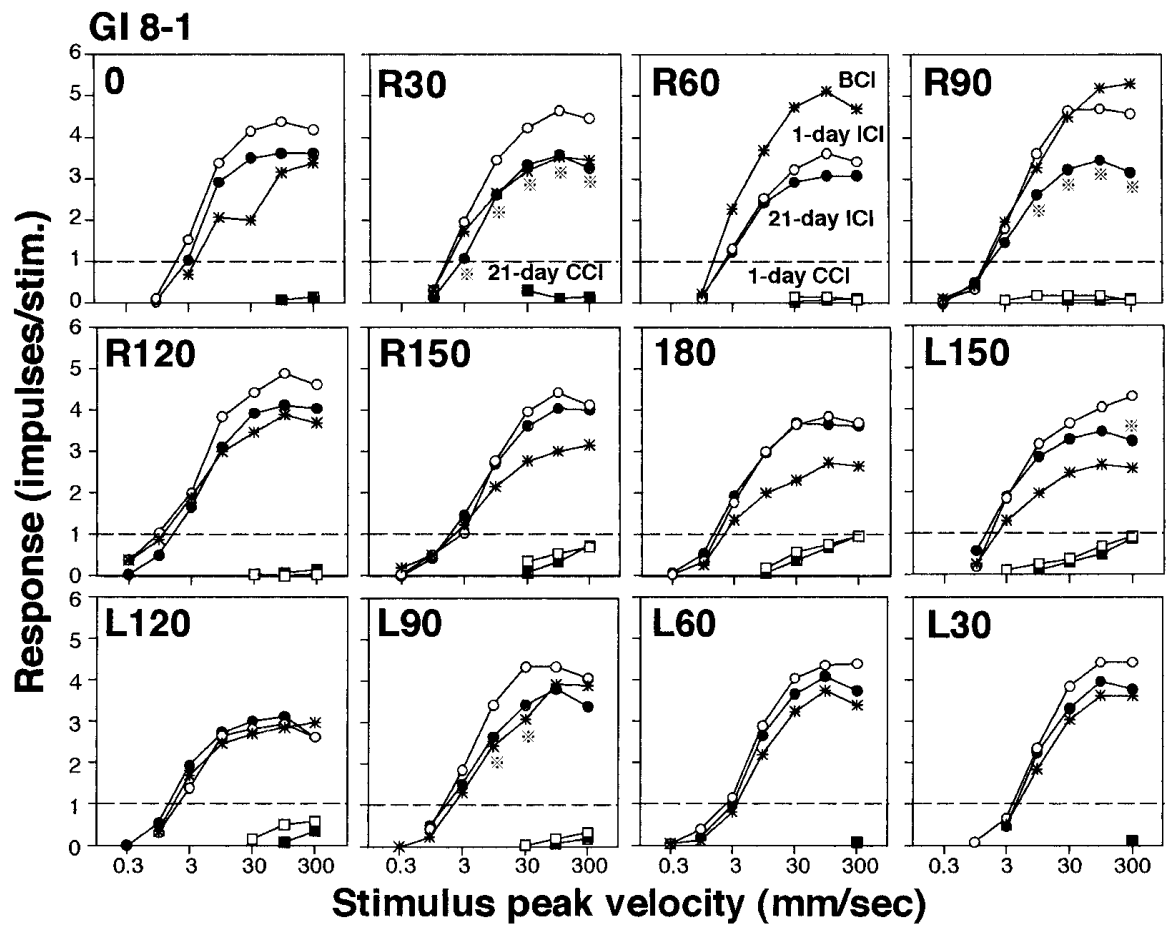

Fig. 2. Intensity-response curves of GI 8-1 measured with a unidirectional air current from 12 different directions. Response magnitudes (averaged number of action potentials elicited per stimulus) were plotted against the peak velocity of the stimulus air current. Symbols are the same as in Fig. 1. ※: statistically significant difference was observed between 1- and 21-day ICl animals. Twenty one days after cercal ablations, most response magnitudes became smaller than those in 1-day animals. Statistically significant cmpensational changes in ICl animals were observed when air currents were applied from R30, L150 and L90 directions. Faint responses observed in 21-day CCl animals were likely to have been caused by the rebound depolarization. $\mathrm{N}$ (number of animals) $=10$ for open circles and filled squares. $\mathrm{N} \geq 10$ for filled circles. $\mathrm{N}=5$ for open squares. 


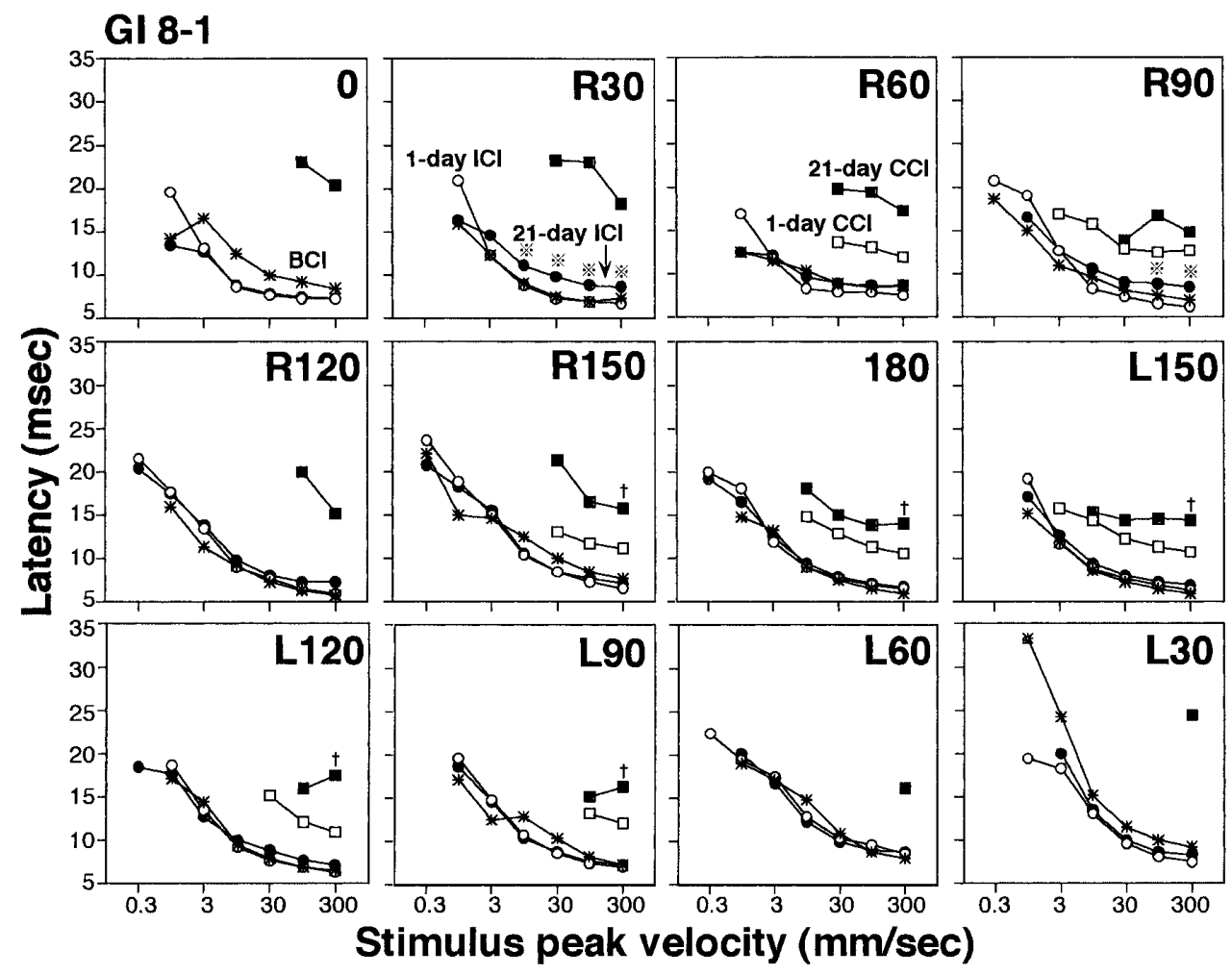

Fig. 3. Response latencies of GI 8-1. Symbols and sample numbers are the same as in Fig. 2. †: statistically significant difference was observed between 1- and 21-day $\mathrm{CCl}$ animals.

tions (Matsuura and Kanou, 1998). However, these stimuli elicited responses on GI 8-1, though very weakly, 21 days after the ablation (Fig. 2). These responses were likely to be caused by the rebound depolarization from an inhibitory hyperpolarization (see discussion).

Response latencies of $\mathrm{Gl}$ 8-1 in 21-day $\mathrm{CCl}$ animals were significantly longer than those in 1-day $\mathrm{CCl}$ animals when the $300 \mathrm{~mm} / \mathrm{sec}$ air current was applied from R150, 180, L150, L120 and L90 directions ( $P<0.05$; Fig. 3). However, none of these changes were compensational. The changes in response latency were likely to be caused by a change of the duration of a hyperpolarization (see discussion).

\section{GI 9-1 in ICl animals}

Velocity thresholds of GI 9-1 in 21-day ICI animals showed no compensational change at all. Therefore, the receptive field of GI 9-1 in such animals was almost identical to that in 1-day $\mathrm{ICl}$ animals (Fig. 1B).

After the initial 20 days, response magnitudes of GI 9-1 in $\mathrm{ICl}$ animals decreased to some extent regardless of the stimulus direction except for the 180 direction (Fig. 4). Among the changes, the ones that occurred when the air currents were applied from R150 and L30 directions were likely to be compensational. However, only a change observed at L30 was statistically significant $(P<0.05$; Fig. 4).

Changes in response latencies of GI 9-1 in 21-day ICI animals seemed compensational when the air currents were applied from R150 to L60 directions (clockwise) (Fig. 5). Among them, however, only the changes to the air current applied from R150 direction were statistically significant and compensational $(P<0.05$; Fig. 5).

\section{GI 9-1 in $\mathrm{CCl}$ animals}

GI 9-1 in 1-day $\mathrm{CCl}$ animals showed no response regardless of the stimulus direction (Matsuura and Kanou, 1998). Like 1-day animals, no response from the Gl was observed even in 21-day $\mathrm{CCl}$ animals.

\section{GI 9-2 in ICl animals}

In 21-day ICl animals, velocity thresholds of GI 9-2 were slightly higher than those in 1-day animals regardless of the stimulus direction (Fig. 1C). However, the differences were not statistically significant and the receptive field in 21-day animals was almost the same as that in 1-day animals.

Response magnitudes of GI 9-2 in 21-day $\mathrm{ICl}$ animals showed statistically significant decrease from those in 1-day $\mathrm{ICl}$ animals when the stimulus air currents were applied from R90, R120, R150, L150, L90, L60 and L30 directions $(P<0.05$; Fig. 6). Among them, changes to the R150, L30 and L150 air currents were likely to be compensational. As the responses to the L30 air current seemed to be caused by a rebound motion of cercal filiform hairs (Matsuura and Kanou, 1998), changes in the response magnitudes to the L30 air current observed here must have been caused by the changes to the 


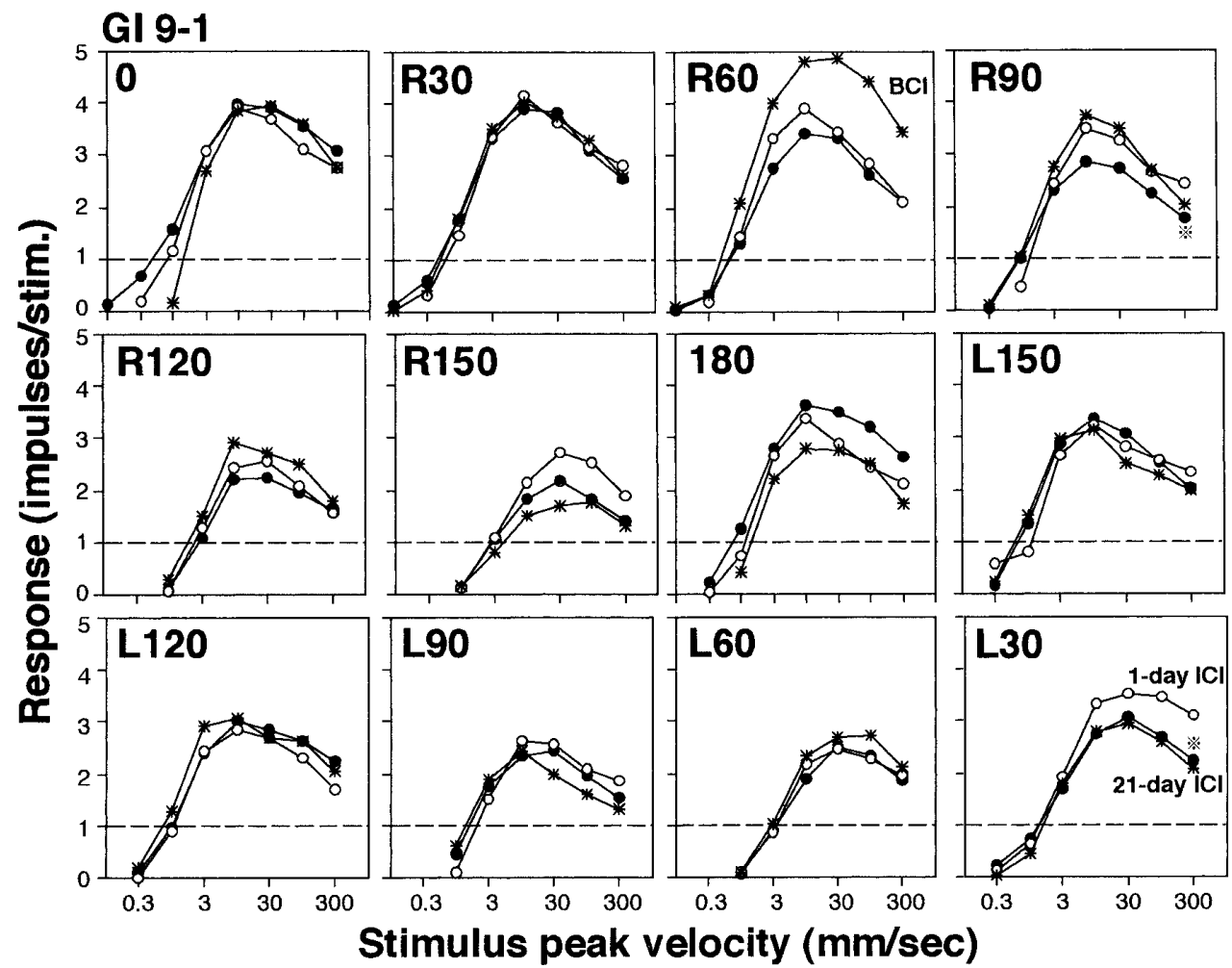

Fig. 4. Intensity-response curves of GI 9-1. Symbols are the same as in Figs. 1 and 2. In ICl animals, statistically significant cmpensational changes in response magnitude were observed when the stimulus air current was applied from L30 direction. In CCl animals, any neural response was not observed even in 21-day animals. $\mathrm{N} \geq 9$ for open and filled circles.

GI 9-1

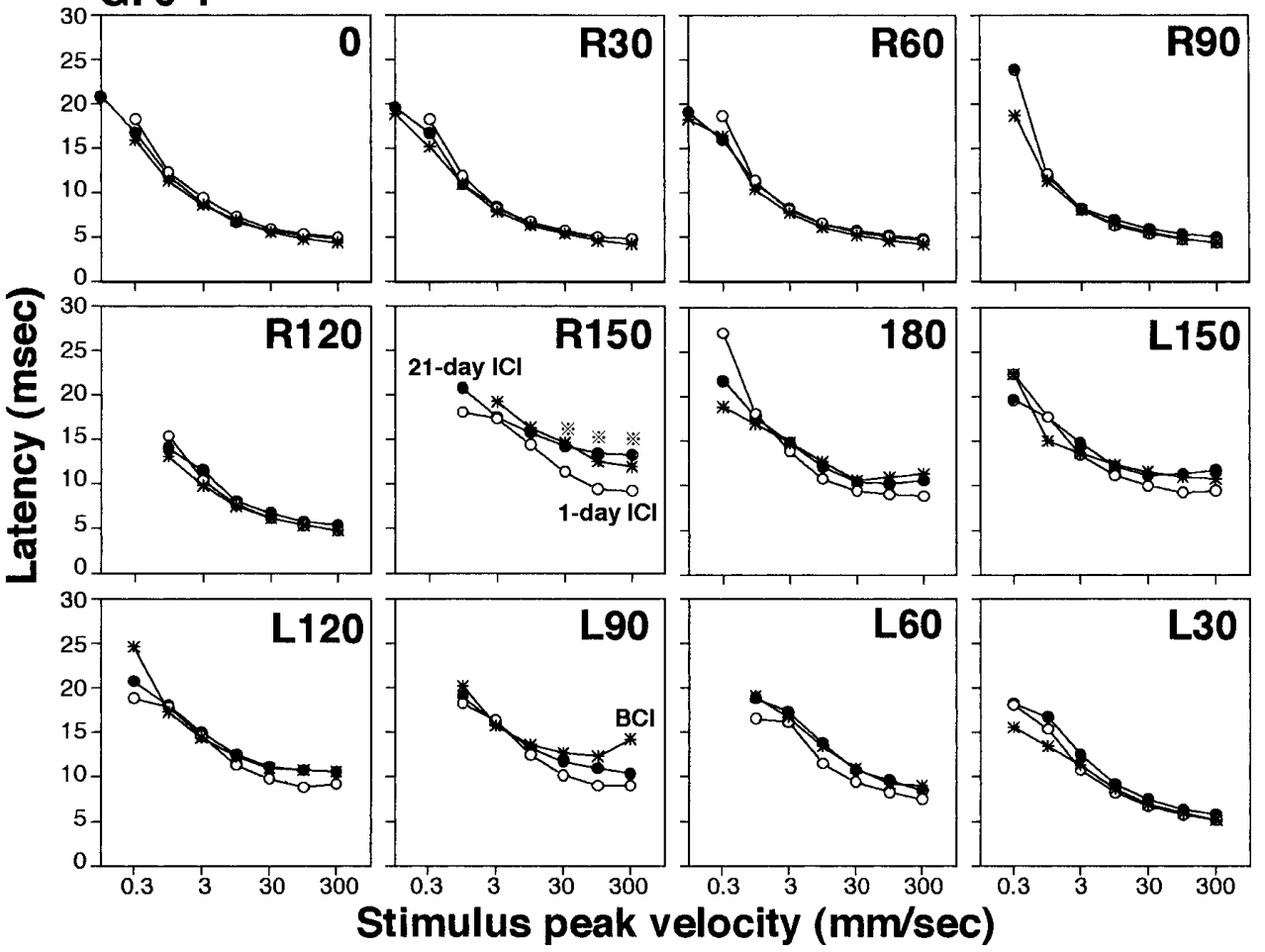

Fig. 5. Response latencies of GI 9-1. Symbols are the same as in Figs. 1 and 2. In ICl animals, statistically significant compensational changes in response latency were observed when the stimulus air current was applied from R150 directions. Sample numbers are the same as in Fig. 4. 


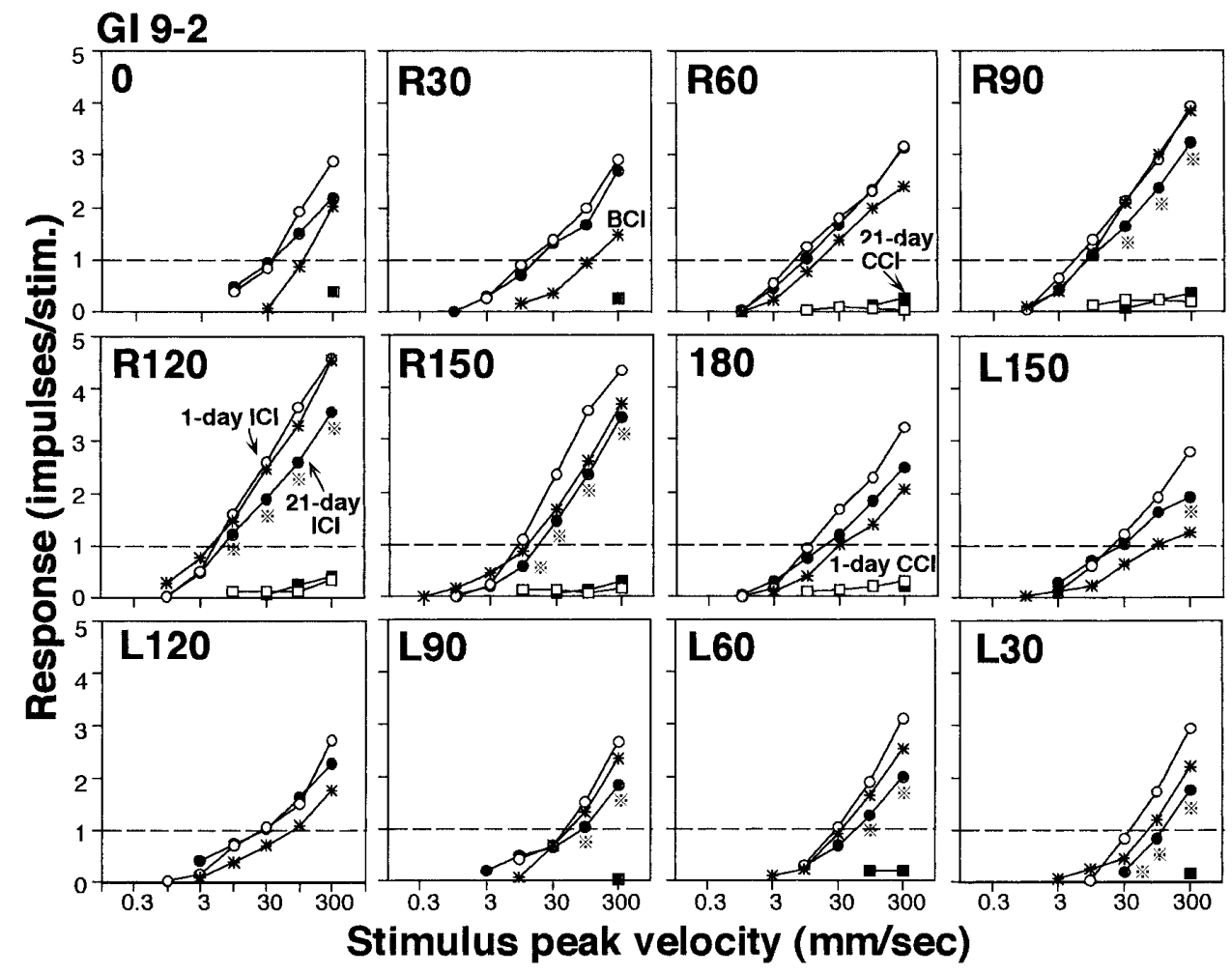

Fig. 6. Intensity-response curves of GI 9-2. Symbols are the same as in Figs. 1 and 2. In ICl animals, typical compensational changes were observed when the air current was applied from R150 direction. Faint responses in 21-day CCl animals to the air currents from 0, R30 and L30 directions were likely to have been caused by the rebound depolarization. $N=10$ for open circles. $N \geq 9$ for filled circles. $N=8$ for open and filled squares.

air current from $180^{\circ}$ opposite direction, i.e. R150. Therefore, in GI 9-2 in 21-day ICl animals, compensational changes in response magnitude were confirmed when the stimulus air currents were applied from R150 and L150 directions.

Response latencies of GI 9-2 in 21-day ICl animals were significantly longer than those in 1-day animals when the stimulus air currents were applied from R90, R120, R150, 180, L150 and $L 30$ directions $(P<0.05 ;$ Fig. 7). Among them, changes to the R120 and R150 air currents were compensational. Most changes in response latency were coupled with the changes in response magnitude, i.e. when the response magnitude became smaller, latencies of the responses became longer in most cases.

\section{GI 9-2 in $\mathbf{C C l}$ animals}

Gl 9-2 in 1-day $\mathrm{CCl}$ animals did not show any response to the air currents from 0, R30, L90, L60 and L30 directions (Matsuura and Kanou, 1998). However, though very weakly, the $\mathrm{Gl}$ in 21-day $\mathrm{CCl}$ animals responded to such stimuli when the velocity of the air current was relatively high (Fig. 6). Like GI 8-1, responses to the air currents from $0, R 30$ and L30 were assumed to be caused by an increase of the amplitude of rebound depolarization (see discussion).

\section{GI 9-3 in ICl animals}

In 21-day ICl animals, velocity thresholds of GI 9-3 were significantly higher than those in 1-day animals when the stimulus air currents were applied from 0, R30, R60 and R90 directions ( $P<0.05 ;$ Fig. 8). Therefore, the receptive field of the Gl in 21-day $\mathrm{ICl}$ animals was smaller than that in 1-day animals (Fig. 1D). However, the changes were not compensational.

During the first 20 days, most response magnitudes of $\mathrm{Gl}$ $9-3$ in $\mathrm{ICl}$ animals decreased to some extent (Fig. 8). Statistically significant changes were observed when the stimulus air currents were applied from $0, R 30$ and $R 60$ directions $(P<$ 0.05 ; Fig. 8). However, the changes were not compensational because differences between intact and treated animals became larger than those in 1-day animals.

Response latencies of GI 9-3 in 21-day ICl animals were slightly different from those in 1-day animals. However, the changes were not statistically significant. No sign of change in response latency was, therefore, ascertained in Gl 9-3 in 21-day ICl animals.

\section{GI 9-3 in $\mathrm{CCl}$ animals}

Velocity thresholds of GI 9-3 in 21-day CCl animals became higher than those in 1-day animals but the changes were not compensational. The receptive field of the Gl became rather smaller during the first 20 days. As no response of the GI was observed when the air currents were applied from contralateral side, the receptive field obtained was restricted to the ipsilateral side (Fig. 1E). 


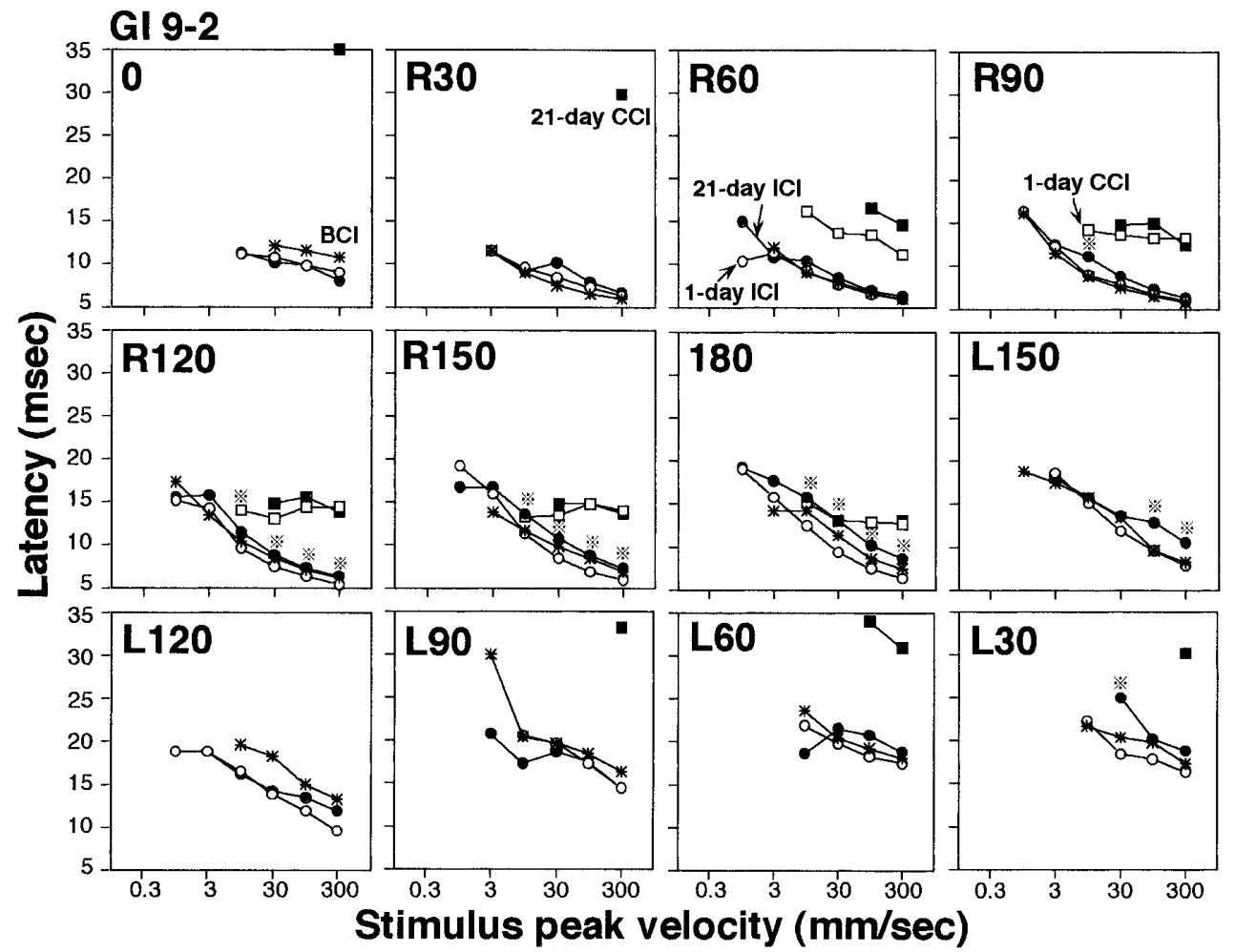

Fig. 7. Response latencies of GI 9-2. Symbols are the same as in Figs. 1 and 2. In ICl animals, statistically significant compensational changes in response latency were observed when the stimulus air current was applied from R120 and R150 directions. Sample numbers are the same as in Fig. 6.

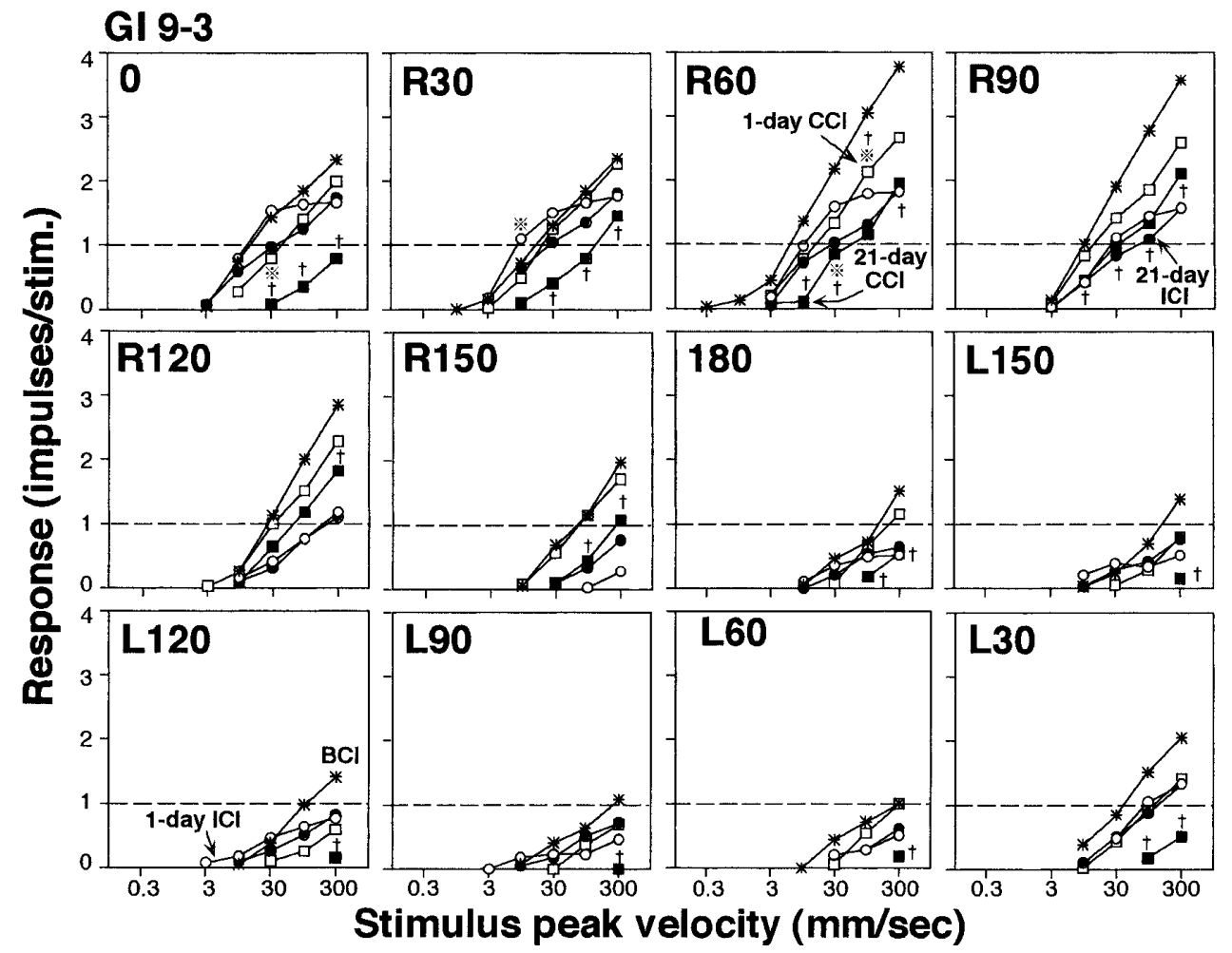

Fig. 8. Intensity-response curves of GI 9-3. Refer to Figs. 1, 2 and 3 for symbols. Although statistically significant changes in response magnitude were frequently observed between 1 - and 21 -day animals, none of them were compensational. $\mathrm{N} \geq 10$ for all. 


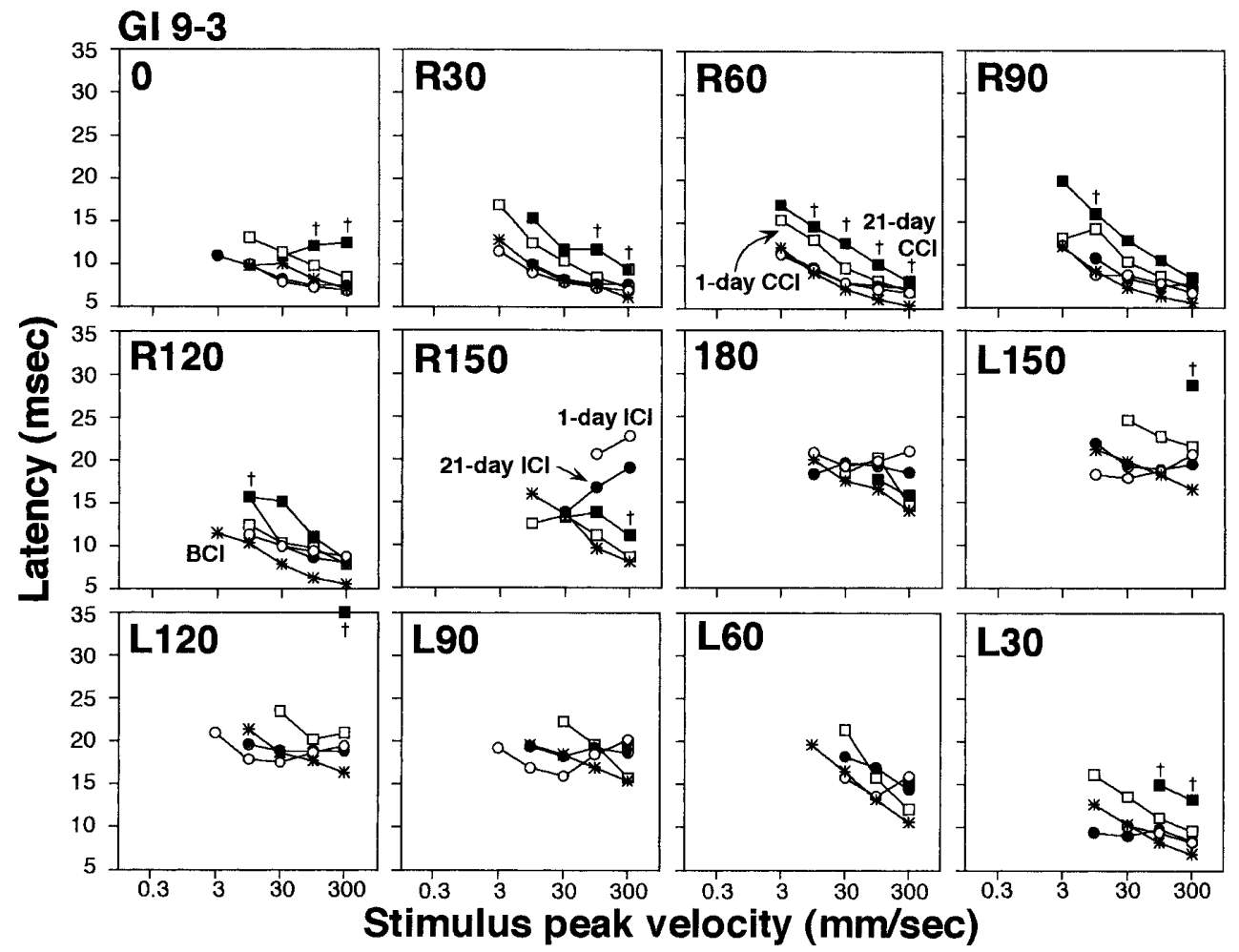

Fig. 9. Response latencies of GI 9-3. Refer to Figs. 1 and 3 for symbols. Response latencies in 21-day $\mathrm{CCl}$ animals were significantly longer than those in 1-day animals. However, the changes were not compensational. Sample numbers are the same as in Fig. 8.

Most response magnitudes of GI 9-3 in 21-day CCl animals decreased significantly compared to those in 1-day animals regardless of the stimulus direction $(P<0.05$; Fig. 8). The Gl showed only very poor responses to the air currents especially from contralateral side as mentioned above. However, none of the changes in response magnitude of GI 9-3 observed in 21-day $\mathrm{CCl}$ animals was compensational.

Most response latencies of GI 9-3 in 21-day CCl animals became significantly longer than those in 1-day animals $(P<$ 0.05; Fig. 9). However, the changes were not compensational.

All the results suggest that excitatory connection between contralateral cercal filiform hairs and Gl 9-3 became weak during the initial 20-day period. As in the case of $\mathrm{ICl}$ animals, no response property of Gl 9-3 in 21-day CCl animals showed any compensational change.

\section{DISCUSSION}

\section{Compensational functional changes in Gls}

In the present study, we showed that some of the Gls in adult crickets made compensational functional changes after unilateral cercal ablations. However, changes were observed only in limited cases (Fig. 10) than we have assumed from the results of our behavioral investigations; i.e. both response rate and orientation of escape to an air-puff stimulus showed relatively large compensational changes during 1 or 2 weeks after the unilateral cercal ablations (Kanou et al., in preparation). One reason for such inconsistency between the results
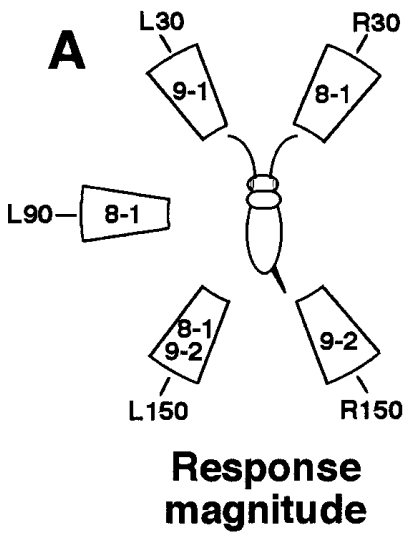

Fig. 10. Gls which showed statistically significant compensational changes to an unidirectional air current and the directions of the stimulus are summarized. (A) response magnitude. (B) response latency. Compensational changes of Gls were confirmed only in $\mathrm{ICl}$ animals.

of neurophysiological and behavioral investigations might be the experimental method which we employed in the present study. The spike count analysis, which we employed in the present study, might not be sensitive enough to detect the faint physiological changes in neurons as a direct measurment of membrane potentials. However, in order to trigger or control the escape behaviors, the air current information integrated in the terminal abdominal ganglion must be encoded in the number of spikes of particular interneurons when sent to other parts of the central nervous systems. Therefore, the response 
magnitude of a GI must show a significant change if the information carried by the Gl is essential for triggering or controlling the escape behaviors.

\section{Direction dependency of functional change in Gls}

Some of the changes in Gls observed 21 days after the unilateral cercal ablation were compensational and some were not. One of the significant features of such functional changes was that they did not appear equally to air currents from different directions, i.e. these changes were direction dependent. For example, compensational changes in the response magnitude of GI 8-1 in ICl animals were observed only when stimulus air currents were from R30, L90 and L150 directions (Fig. 10). In other Gls, changes in the response magnitude and/or response latency were also direction dependent (Fig. 10). These results suggest that characteristic changes of the neural connection occurred somewhere in the neural pathways between sensory afferents of cercal filiform hairs with particular directionalities on the remaining cercus and a particular GI, because no regeneration of ablated cercus or cercal filiform hairs have been observed during the first 20 days.

\section{Different ways of functional change after unilateral cercal ablation}

We showed how the responses of each Gl changed 21 days after the unilateral cercal ablation. At first, we expected that potentiation of excitatory connection would occur frequently. Only the changes observed in response magnitudes of $\mathrm{Gl} 9-1$ in $\mathrm{ICl}$ animals to $180^{\circ}$ stimulation were likely to be caused by the potentiation because response magnitudes in 21-day animals were larger than those in 1-day animals. The differences, however, were not statistically significant. Therefore, no sign of the potentiation of excitatory connection was detected in any GI by the spike count analysis employed in the present study. Changes occurred during the first 20 days seemed to be categorized into the following 2 types: the fading of excitatory connections, or the potentiation of inhibitory connections.

In all the Gls, most response magnitudes in 21-day $\mathrm{ICl}$ animals (and also in $\mathrm{CCl}$ animals for Gl 9-3) were smaller than those in 1-day animals. Such changes are likely to be caused by the formation of new inhibitory neural pathways or by the fading of excitatory connections. In our previous study, though it was a spike count analysis, no inhibitory connection between ipsilateral filiform hairs and any Gls was confirmed, i.e. inhibitory information were only from the contralateral filiform hairs in Gls 8-1, 9-1 and 9-2 (inhibitory input has not been confirmed in GI 9-3 at all even from the contralateral filiform hairs) (Matsuura and Kanou, 1998). In addition, no regeneration of ablated cercus or cercal filiform hairs was observed during the 21 days because we used adult crickets after the final molt. Based on these facts, it could be confirmed that the changes were caused by the fading of excitatory connections rather than the formation of new inhibitory neural pathways.

In 1-day $\mathrm{CCl}$ animals, Gls 8-1 and 9-2 did not show any response to the air currents applied from particular directions; i.e. 0, R30, L30 and L60 for GI 8-1, and 0, R30, L30, L60 and L90 for GI 9-2. However, the Gls showed very faint responses to those air currents after 20 days (Figs. 2, 6). The responses, other than to L60 and L90 air currents in GI 9-2, were not likely to have been caused by the formation of new excitatory connections but were likely to have been caused by the potentiation of rebound depolarization after the release from inhibitory hyperpolarization. The reasons are: 1) response magnitudes were very small (Figs. 2, 6); 2) response latencies were very long (Figs. 3, 7); and 3) air currents from those directions showed inhibitory effects on the Gls in 1-day animals (Matsuura and Kanou, 1998). It is unlikely that the inhibitory connections changed to excitatory ones during the first 20 days. Therefore, we assumed that the responses were caused by a potentiation of inhibitory connections. The hyperpolarization must have become larger during the 20 days, and the release from such larger hyperpolarization must have resulted in larger rebound depolarization that could elicit neural responses.

Changes in response latencies in Gl 8-1 might be another example of the change in inhibitory effects. In 21-day $\mathrm{CCl}$ animals, response latencies of GI 8-1 to the $300 \mathrm{~mm} / \mathrm{sec}$ air currents were significantly longer than those in 1-day $\mathrm{CCl}$ animals when the air currents were applied from R150, 180, L150, L120 and L90 directions ( $P<0.05$; Fig. 3). In 1-day CCI animals, responses to these air currents were assumed to be caused by the rebound depolarization after the inhibition (Matsuura and Kanou, 1998). Therefore, the duration of the inhibitory hyperpolarization was likely to become longer during the 20 days and resulted in longer latency responses caused by the rebound depolarization.

The potentiation of inhibition in $\mathrm{CCl}$ animals may suggest that the synaptic competition (Guillery, 1972) of excitatory and inhibitory inputs regulates the response properties of the Gls. For example, as all the ipsilateral inputs on GI 8-1 are excitatory, the long-term absence of such excitatory inputs in $\mathrm{CCl}$ animals made the inhibitory effect from contralateral cercal filiform hairs more powerful and resulted in larger and longer hyperpolarizations as mentioned above.

\section{ACKNOWLEDGMENT}

The authors are grateful to Dr. B. A. Ardekani, Department of Radiology and Nuclear Medicine, Akita Research Institute of Brain and Blood Vessels, for his critical reading and English proof of the manuscript. This work was partially supported by a Grant-in-Aid for Scientific Research 07640903 from the Japanese Ministry of Education, Science, Sports and Culture to M. K.

\section{REFERENCES}

Carew TJ, Sahley CL (1986) Invertebrate learning and memory: from behavior to molecules. Annu Rev Neurosci 9: 435-487

Fetz E, Baker MA (1973) Operantly conditioned patterns of precentral unit activity and correlated responses in adjacent cells and contralateral muscles. J Neurophysiol 36: 179-204

Gonshor A, Melvill-Jones GM (1976) Extreme vestibulo-ocular adap- 
tation induced by prolonged optical reversal of vision. J Physiol (Lond) 256: 381-414

Guillery RW (1972) Binocular competition in the control of geniculate cell growth. J Comp Neur 144: 117-130

Ito M (1982) Cerebellar control of the vestibulo-ocular reflex - around the flocculus hypothesis. Ann Rev Neurosci 5: 275-296

Kandel ER (1979) Cellular insights into behavior and learning. The Harvey Lectures 73: 19-92

Kanou M (1991) Threshold and directional sensitivity of air-current sensitive giant interneuron of a cricket. Experientia 47: 447-448

Kanou M (1996) Directionality of cricket giant interneurons to escape eliciting unidirectional air-current. Zool Sci 13: 35-46

Kanou M, Shimozawa T (1984) A threshold analysis of cricket cercal interneurons by an alternating air current stimulus. J Comp Physiol A 154: 357-365

Kanou M, Ohshima M, Inoue J (1995) Analysis of air-current evoked escape behavior of the cricket. Zool Sci 12 (Suppl): 100 (Proceedings of the 66th annual meeting of the Zoological Society of Japan)
Matsuura T, Kanou M (1998) Organization of receptive fields of cricket giant interneurons revealed by cercal ablations. Zool Sci 15: 183194

Shimozawa T, Kanou M (1984a) Varieties of filiform hairs: range fractionation by sensory afferents and cercal interneurons of a cricket. J Comp Physiol A 155: 485-493

Shimozawa T, Kanou M (1984b) The aerodynamics and sensory physiology of range fractionation in the cercal filiform sensilla of the cricket Gryllus bimaculatus. J Comp Physiol A 155: 495-505

Thompson RF (1986) The neurobiology of learning and memory. Science 233: 941-947

Tinbergen N (1969) The Study of Instinct. Clarendon Press, Oxford

Tsukahara N (1981) Synaptic plasticity in the mammalian central nervous system. Ann Rev Neurosci 4: 351-379

Vardi N, Camhi JM (1982) Functional recovery from lesions in the escape system of the cockroach. 1. Behavioral recovery. J Comp Physiol A 146: 291-298

(Received May 22, 1997 / Accepted January 29, 1998) 